Instituto Internacional de Investigación y Desarrollo Tecnológico Educativo INDTEC, C.A.

DOI: https://doi.org/10.29394/Scientific.issn.2542-2987.2018.3.7.9.177-192

OAI-PMH: http://www.indteca.com/ojs/index.php/Revista Scientific/oai

\title{
Motivación Laboral. Elemento Fundamental en el Éxito Organizacional
}

\author{
Autores: Humberto Coromoto Peña Rivas \\ Universidad Estatal Península de Santa Elena, UPSE \\ hpenari@upse.edu.ec; hcpr100271@gmail.com \\ La Libertad, Ecuador \\ Sabina Gisella Villón Perero \\ Universidad Estatal Península de Santa Elena, UPSE \\ svillonp@upse.edu.ec; sabinagisella@hotmail.es
}

La Libertad, Ecuador

\section{Resumen}

El presente estudio tiene como objetivo analizar la influencia de la motivación en el talento humano y determinar los factores que tienen mayor impacto en el desempeño laboral. La investigación se desarrolló en base a una metodología hermenéutica, que inició en la revisión bibliográfica-documental permitiendo obtener las referencias teóricas, criterios básicos y relevantes acerca de la motivación laboral, de tal manera que la obtención, identificación y descripción de los datos facilitó el logro del objetivo planteado, obteniendo como resultado que un empleado motivado brindará a la empresa un mejor desempeño, desarrollará sentido de pertenencia, fidelidad a lo que hace, reflejando como consecuencia beneficios tanto para la empresa como para los empleados concluyendo que el comportamiento de un empleado en la organización, depende de los factores motivacionales que se apliquen a fin de satisfacer sus necesidades básicas como son las buenas relaciones laborales, la satisfacción laboral, clima laboral positivo y los resultados de la satisfacción laboral; actualmente, motivar de manera acertada se ha convertido en una actividad que logrará que los empleados creen un sentido de pertenencia y la actividad que realiza cada uno de ellos, arroje resultados positivos a fin de contribuir con éxito en lo que hace la organización. Como elemento fundamental en el desarrollo asertivo de la organización, la motivación guarda una estrecha relación con la satisfacción laboral, las relaciones laborales y el entorno laboral, sin dejar de lado al rol del gerente quien juega un papel especial, al momento de llevar la responsabilidad de dirección y de aplicar las estrategias más adecuadas de motivación dentro de una organización empresarial.

Palabras clave: motivación; recursos humanos; organización. 


\title{
Labor Motivation. Fundamental Element in Organizational Success
}

\begin{abstract}
The present study aims to analyze the influence of motivation on human talent and determine the factors that have the greatest impact on work performance. The research was developed on the basis of a hermeneutic methodology, which began in the bibliographical-documentary revision allowing to obtain the theoretical references, basic and relevant criteria about the labor motivation, in such a way that the obtaining, identification and description of the data facilitated the achievement of the objective set, resulting in a motivated employee to provide the company with a better performance, develop a sense of belonging, fidelity to what it does, reflecting as a consequence benefits for both the company and employees concluding that the behavior of an employee in the organization, depends on the motivational factors that are applied in order to satisfy their basic needs such as good labor relations, job satisfaction, positive working environment and the results of job satisfaction; nowadays, motivating rightly has become an activity that will get employees to create a sense of belonging and the activity that each of them perform, yield positive results in order to contribute successfully in what the organization does. As a fundamental element in the assertive development of the organization, motivation is closely related to job satisfaction, labor relations and the work environment, without neglecting the role of the manager who plays a special role, at the time of carrying the responsibility and apply the most appropriate strategies of motivation within a business organization.
\end{abstract}

Keywords: motivation; human resources; organization. 


\section{Introducción}

Mucho se ha hablado sobre la motivación y es que actualmente la motivación es un elemento que se encuentra presente en todos los momentos de la vida del ser humano; así diversas ramas de la ciencia la han conceptualizado. Para la Psicología la motivación se define como la necesidad o el deseo que activa y dirige nuestro comportamiento, que lo dirige y subyace a toda tendencia por la supervivencia. Así mismo surgen diversas teorías que tratan de analizar la esencia intrínseca acerca de la motivación y sus teorías y establecer ¿Cómo la motivación despierta al ser humano a fin de suplir sus necesidades?

Actualmente a nivel organizacional se presentan un sinfín de escenarios que conllevan para tener en cuenta la rapidez con la que fluyen las nuevas tecnologías, la globalización, el permanente cambio del contexto y la valoración del conocimiento, tomando en cuenta que los tiempos actuales están sujetos a cambios drásticos y exponenciales que dan paso a la realización de procesos de cambio en secuencia dentro de las organizaciones empresariales.

Cabe destacar que, como elemento fundamental en el desarrollo asertivo de la organización, la motivación guarda una estrecha relación con la satisfacción laboral, las relaciones laborales y el entorno laboral. Todas las empresas que mantienen un alto grado de motivación en sus empleados también tendrán un alto grado de satisfacción hacia sus clientes.

El presente artículo analiza cómo influye la motivación en el talento humano para lograr el éxito organizacional. Saber motivar a los empleados se ha convertido en un rasgo de éxito para las organizaciones ya que de ello dependerá mayor producción laboral. Así mismo plasmar un resultado expreso, aludiendo algunas teorías de la motivación que se han desarrollado a lo largo del tiempo y crear una visión objetiva ante el uso de estas teorías en las organizaciones empresariales. 
Con el presente análisis surgen las siguientes interrogantes: ¿Cómo influye la motivación en el desempeño laboral del individuo en la organización empresarial? ¿Cuáles son los elementos que intervienen y que tienen mayor impacto sobre el desempeño, la motivación y la calidad en el trabajo? Así mismos analizar los siguientes aspectos que guardan relación entre la satisfacción laboral y objetivos organizacionales; elementos motivadores del talento humano y las diversas teorías existentes.

Es así como se infiere que la teoría de Herzberg, se manifiesta en ver al empleado como el ser que busca el reconocimiento dentro de la organización y la satisfacción de sus necesidades y que al satisfacer estos dos objetivos, su motivación se convertirá en el impulsador para asumir responsabilidades y encaminar su conducta laboral a lograr metas que permitirán a la organización lograr con éxito su razón de ser, con altos niveles de eficacia.

El propósito principal del presente estudio es analizar la influencia de la motivación en el talento humano para lograr el éxito organizacional y determinar los elementos que tienen mayor impacto en el desempeño, la motivación y la calidad en el trabajo.

\section{Desarrollo}

Desde la invención de la máquina hasta la actualidad, el avance de las tecnologías, la competitividad y el crecimiento organizacional de las empresas son elementos que van a lo largo de los años incrementando su desarrollo productivo a fin de garantizar el éxito corporativo. Pero ese éxito corporativo va más allá de la venta de un producto. Se trata más bien de cómo influye el factor humano en el proceso de producción desde la perspectiva de la motivación a los empleados.

Vivimos en una era en la que el gerente juega un papel especial, al momento de llevar la responsabilidad de dirección dentro de una organización 
empresarial. Dirigir una organización no es tarea sencilla puesto que cada individuo posee una identidad única que lo conduce a ostentar un criterio propio o un pensamiento intrínseco en su actuar. Es en ese momento que comienza la actividad del gerente. El gerente debe influir y motivar a sus empleados de tal manera que cada actividad laboral resulte provechosa y exitosa.

Actualmente, el motivar acertadamente a los empleados se ha convertido en una actividad que logrará que los empleados creen un sentido de pertenencia y la actividad que realiza cada uno de ellos, arroje resultados positivos a fin de contribuir con éxito en lo que hace la organización.

Generalmente, el éxito organizacional se construye con esfuerzo y dedicación, y para lograrlo la motivación constituye una meta concreta en el arduo trayecto hacia el éxito, y este logro ocasiona un desgaste enérgico para el trabajador.

En la actualidad las organizaciones invierten grandes sumas de dinero con el fin aplicar estrategias que motiven a su personal a realizar mayores esfuerzos con la intención de conquistar logros para la organización, obviando en algún momento las necesidades de cada trabajador, y es en este momento donde esa inversión puede resultar en fracaso; ya que estas necesidades son de carácter individual y varían en el tiempo. Sin embargo, esas necesidades son el proceso que activa el comportamiento, y en algunos casos pueden ser semejantes en todos los trabajadores, es decir, aunque sus patrones de comportamiento varíen, el proceso que los motiva será el mismo para todos los trabajadores.

\subsection{La motivación}

Para entender todos los aspectos que se abordarán en referencia a la motivación laboral, es necesario partir desde la comprensión conceptual de la motivación. Existen un sin número de definiciones que abordan la motivación 
y que vienen a ser el producto de diferentes estudios que se han realizado al presente tema. Con la intención de sustentar el presente artículo, se citan algunas definiciones:

Chiavenato (2000a: 68), afirma que la motivación se relaciona con la conducta humana, y es una relación basada en el comportamiento en donde éste es causado por factores internos y externos en los que el deseo y las necesidades generan la energía necesaria que incentiva al individuo a realizar actividades que lograrán cumplir su objetivo.

Delgado (1999: 56), define la motivación como el proceso mediante el cual las personas, al ejecutar una actividad específica, resuelven desarrollar unos esfuerzos que conduzcan a la materialización de ciertas metas y objetivos a fin de satisfacer algún tipo de necesidad y/o expectativa, y de cuya mayor o menor satisfacción van en dependencia del esfuerzo que determinen aplicar a futuras acciones.

González (2008: 52), puntualiza la motivación como un conjunto de procesos psíquicos que vinculada con los estímulos externos mantiene un intercambio con la personalidad que a la vez es influenciada por estos, generando o no satisfacción.

Tal como se han planteado algunas definiciones propuestas por diferentes autores, destacan el origen de la motivación en el individuo y convergen en que la necesidad es el punto de partida para generar la actitud necesaria y plantearse metas que generen el éxito. Sin embargo, una vez cubierta la necesidad, el individuo creará otras necesidades que continúen motivando las metas que desee alcanzar.

\subsection{Teorías de la Motivación}

En este sentido, surgen diversas de teorías sobre la motivación las cuales tratan de explicar y definir la motivación y que vienen a contribuir con la búsqueda incesante sobre la satisfacción de las necesidades humanas, 
entre ellas destacan:

\subsubsection{Teoría Jerarquía de las necesidades de Maslow}

Maslow (1964), clasificó la motivación humana orientándola hacia una perspectiva intrínseca inherente al individuo, jerarquizando estas necesidades en dos categorías: Categoría necesidades primarias, en las que clasifica dos niveles; el primer nivel: necesidades fisiológicas tales como: alimentación, sueño y reposo, abrigo o deseo sexual; y el segundo nivel: necesidades de seguridad en las que se busca proteger contra amenaza o peligro; denominándolas necesidades básicas que vienen a garantizar la supervivencia y seguridad del individuo. Y la Categoría necesidades secundarias: clasificadas en tres niveles, el primer nivel: necesidades sociales se encuentran ligadas a la forma de actuar del individuo en sociedad, donde se involucran el afecto, la aceptación, la participación, la amistad y el amor; el segundo nivel: las necesidades de autoestima, es la valoración que el propio individuo realiza de sí mismo. $\mathrm{Y}$ en el tercer nivel: las necesidades de autorrealización: en las que se conduce al individuo a desarrollar su máximo potencial y a superarse como seres humanos.

\subsubsection{Teoría de los dos Factores de Herzberg}

Chiavenato (2000b), clasificó la motivación en dos factores orientados al ambiente externo y al trabajo del individuo. El primer factor: Higiénico: compuesto por las condiciones físicas y ambientales de trabajo, es decir, las que rodean al individuo y comprenden: salario, beneficios sociales, políticas de la empresa, clima de las relaciones laborales. Así mismo corresponden a las oportunidades existentes, a la perspectiva ambiental y a los mecanismos que las organizaciones realizan para motivar a los empleados. El segundo factor: Motivacional: relacionado con todas las actividades inherentes al cargo tales como: la realización de un trabajo importante, el logro, la responsabilidad, 
ascensos, reconocimientos, utilización de habilidades y que satisfacen en el tiempo y aumentan la productividad organizacional.

\subsubsection{La teoría de McClelland}

McClelland (1970, citado por Perilla, 1998), clasificó la teoría basada en tres necesidades: logro, poder y afiliación. En la que establece que una necesidad insatisfecha origina una tensión que promueve unos impulsos en el interior del individuo. McClelland destacó a su vez tres de estos impulsos más dominantes: La motivación para el logro: el individuo desea crecer y desarrollarse. La motivación por afiliación: relacionarse con las personas en un ambiente social. La motivación por competencia: es un impulso por realizar un trabajo de gran calidad. Y la motivación por poder: es un impulso por influir en las personas y cambiar las situaciones.

\subsubsection{Teoría de la Expectativa de Vroom}

Vroom (1979, citado por Chiavenato, 2000c), basado en la motivación para producir, alega que en cada individuo existen tres factores que determinan la motivación para producir en los que se mencionan los objetivos individuales que poseen la fuerza de voluntad para lograr objetivos; la relación percibida por el individuo entre la productividad y el logro de los objetivos individuales y la capacidad del individuo para influir en su nivel de productividad. La importancia de la presente teoría reposa en el interés que se observa de la individualidad y la variabilidad de las fuerzas motivadoras.

\subsubsection{Teoría erc de Alderfer}

Alderfer (1972), fundamenta sus investigaciones en el modelo de Maslow y plantea tres motivaciones básicas de la siguiente forma: Motivaciones de existencia: conciernen a las necesidades fisiológicas y de seguridad; Motivación de relación: conciernen a las interacciones sociales con 
otros, apoyo emocional, reconocimiento y sentido de pertenencia al grupo; y Motivación de crecimiento: Se concentra en el desarrollo y crecimiento personal. (Robbins, 2004: 128-132).

\subsubsection{Teoría del Estabelecimiento de las Metas}

Locke (1969: 991-1009), afirma que las personas imponen sus propias metas con la finalidad de lograrlas. La presente teoría asevera que trabajar hacia una meta es una base importante en la motivación del trabajo que se realiza, ya que las metas le indican al empleado lo que necesita realizar y el esfuerzo que debe aportar para lograr el objetivo. Si las personas practican el establecimiento de meta, es más probable que se plantee un objetivo difícil porque se sienten más comprometidos con las opciones en las que han intervenido.

\subsection{Motivación Laboral}

Diariamente, los seres humanos experimentan crean miles de necesidades, las cuales vienen a ser ausencia o falta de elementos materiales o necesidades interiores que desean satisfacer. Es de esta forma como la motivación se convierte en un elemento idóneo para suplir esas necesidades. De tal manera que cuando un empleado logra sentirse motivado en su trabajo, éste logrará un mejor desempeño y desarrollará un sentido de pertenencia dentro de la empresa.

La motivación laboral se puede entender como el resultado de la interrelación del individuo y el estímulo realizado por la organización con la finalidad de crear elementos que impulsen e incentiven al empleado a lograr un objetivo. Es la voluntad que caracteriza al individuo a través del esfuerzo propio a querer alcanzar las metas de la organización ajustado a la necesidad de satisfacer sus propias necesidades. Es decir, es el impulso de satisfacer las necesidades que se haya presente en el empleado en la autorrealización, 
mejores condiciones laborales y satisfacción de necesidades personales, vienen a ser el detonante que producirá la reacción en el empleado a fin lograr esa máxima sensación de confort que produce la realización o cristalización de la meta planteada.

\section{Metodología}

El presente artículo se abordó desde la metodología hermenéutica, ya que parte de la experiencia descriptiva de la reflexión bibliográfica para alcanzar los objetivos planteados y una revisión teórica que permitió identificar los aspectos que tienen mayor impacto sobre la motivación, la motivación laboral y la relación existente entre el logro de los objetivos organizacionales y la satisfacción de los empleados. El presente reconocimiento teórico, como propuesta metodológica, contempla la revisión de diversos textos, de carácter científico.

Por lo antes expuesto, el desarrollo de la investigación se inició aplicando un estudio documentado y exploratorio, a partir de una revisión bibliográfica-documental, la misma que permitió el desarrollo del marco conceptual referente al tema de estudio, cuyos resultados se han sintetizado en el presente trabajo investigativo.

En consecuencia, a ello se establecieron parámetros básicos y relevantes, así como también valiosos criterios acerca de la motivación laboral y los principales factores que generan impacto en el desempeño laboral, de tal manera que la obtención, identificación y descripción de los datos resultó ser más factible, facilitando el logro del objetivo planteado.

A partir de ello, se procedió al análisis de la información obtenida, destacando los elementos más relevantes como los principales factores que intervienen en la motivación laboral y la importante participación de los gerentes con respecto a la responsabilidad que tienen para aplicar estrategias que permitan mantener motivado al talento humano y como consecuencia de 
esto, el logro de los objetivos empresariales.

\section{Factores que intervienen en la motivación laboral}

Existen diversos factores que intervienen en el proceso de la motivación laboral y que van a coadyuvar en la satisfacción del logro de las metas propuestas, sean individuales o colectivas. Entre algunos factores se destacan: $1^{\circ}$ Presencia de una relación laboral donde figure la satisfacción, motivación y desempeño; $2^{\circ}$ Predominio de las expectativas y las compensaciones en cuanto a la satisfacción laboral; $3^{\circ}$ Clima laboral positivo en donde el trabajador encuentre el confort; y $4^{\circ}$ Resultados de la satisfacción laboral.

Una vez establecidos estos factores, y la motivación se encuentra presente, se logra estimular al trabajador en su comportamiento, con la finalidad de lograr la meta deseada. Cuando el trabajador haya experimentado esa sensación de haber culminado su meta con éxito, sentirá haber logrado su objetivo y buscará otra necesidad que satisfacer. El trabajador siempre anhela obtener lo que se plantea y las compensaciones vienen a ser el resultado de lo que se obtiene.

\section{Participación de los gerentes}

En todas las organizaciones empresariales los gerentes juegan un papel importante ya que sobre ellos se encuentra la responsabilidad de aplicar mecanismos que logren integrar y motivar a sus empleados. Es una ardua tarea ya que cada empleado lo motiva alguna cosa distinta; lo que se reduce a que el gerente debe conocer las preocupaciones, las expectativas y las necesidades de cada empleado. Así mismo, el gerente debe confiar en los empleados, delegando actividades en las que el empleado siente que confían en él, de esta manera el empleado creará sentido de pertenencia hacia la organización. 
Los gerentes deben aplicar estrategias que permitan establecer la conexión entre el empleado y la alta gerencia. Así mismo el gerente debe exigir, pero también debe reconocer la labor de los trabajadores. La gerencia de recursos humanos debe asumir la responsabilidad de crear mecanismos que permitan evaluar la actitud y el comportamiento de cada empleado, ya que una gestión adecuada permitirá alcanzar las metas en pro de la organización.

Es necesario dirigir adecuadamente a las personas que laboran en la organización, estimular al trabajador a fin de lograr el equilibrio que permita crecer al empleado y lograr su estabilidad y a la empresa lograr su éxito organizacional.

Tal como se ha planteado, se puede observar que la motivación laboral constituye uno de los elementos de gran importancia para que una organización posea el máximo potencial productivo, por lo que se puede afirmar que un jefe debe contar con buenas herramientas que logren desarrollar una eficiente motivación empresarial.

\section{Conclusión}

El acelerado crecimiento de las organizaciones conduce a crear estrategias que generen cambios positivos en las metas propuestas de cada empresa. En la actualidad para la gran mayoría de organizaciones se crean nuevas incertidumbres sobre los procesos motivacionales, lo que conduce a crear nuevas técnicas que permitan estimular al empleado creando nuevos paradigmas con el fin de optimizar los retos que se plantea cada organización y a su vez en procura de ofrecer un óptimo equilibrio empresa-trabajador.

Realizar un análisis sobre la motivación laboral en la actualidad resulta una tarea titánica pues es evidente que existe numerosa información especializada en referencia al tema. Sin embargo, es imperativo innovar conceptos tomando como base las teorías originarias que han coadyuvado en la actualidad a establecer las necesidades del trabajador y a aplicar las 
estrategias que permitan satisfacer esas necesidades en el individuo en función de sus prioridades y las de la organización.

El desafío esencial que afrontan las organizaciones en la actualidad demanda que se apliquen e intensifiquen estrategias que permitan una acertada motivación laboral. El resultado final será la creación de un vínculo significativo los elementos necesarios para establecer la armonía y el equilibrio entre la organización y sus empleados. Asimismo, lograr compensar necesidades básicas, de esta manera el empleado sentirá la satisfacción de ser útil y valorado, sentir que su trabajo es un valor agregado a la organización; creando sentido de pertenencia y a su vez atmosfera de trabajo favorable que establece patrones de conducta, los cuales harán grata la convivencia en el creciente proceso social del individuo.

En cada individuo fluyen necesidades diferentes, de esta misma forma deben ser cubiertas, aun cuando se creen en el entorno laboral, es necesario crear mecanismos dentro de la organización con la intención de suplir inmediatamente estas necesidades, de esta manera se logrará que el empleado trabaje motivado y se sienta parte de la organización para la cual labora; de esta forma se obtienen las respuestas a las interrogantes planteadas, ya que el desempeño laboral de un individuo desmotivado repercute de forma negativa en el éxito de la organización; Así mismo establecer factores que intervengan con mayor impacto sobre el desempeño y la motivación laboral en el empleado creando un método de distinciones y mejoramiento laboral, condiciones favorables en el ambiente de trabajo, las cuales se hacen necesarias para contribuir a mejorar el rendimiento laboral de los empleados en la organización.

Es importante destacar que toda organización empresarial es responsable de crear mecanismos mediadores que permitan crecer al empleado dentro de la organización, reconocer las divergencias de cada empleado a fin de ofrecerle las mejores soluciones motivacionales tales como 
incentivos, elogios, ascensos, aumentos; de tal forma que el empleado logre sentirse cómodo y satisfecho en su ambiente laboral, que sienta que se le toma en cuenta y que se le brindan oportunidades para surgir, de esta manera el empleado será más productivo y se sentirá con mayor disposición de cooperar en todas las actividades que la organización requiera.

\section{Referencias}

Alderfer (1972). Teorías de motivación; teoría de jerarquía de Alderfer. Recuperado de: https://teoriasmotivacionales.wordpress.com/teoriasmodernas-de-motivacion/teoria-de-jerarquia-de-alderfer/

Chiavenato, I. (2000a,b,c). Administración de Recursos humanos. Bogotá, Colombia: Editorial McGraw-Hill. Quinta edición.

Delgado, N. (1999). Los Estilos de Enseñanza en Educación Física. Universidad de Granada, Granada.

González, S. (2008). Psicología de la motivación. La Habana: Editorial Ciencias Médicas.

Locke, A. (1969). Purpose whitout consciousness: a contradiction. Psychological reports.

Maslow, A. (1964). Religions, Values and Peak-experiences. Columbus, $\mathrm{OH}$ : Ohio State University Press.

Perilla, L. (1998). David C. McClelland (1917-1998). Revista Latinoamericana de Psicología, volumen 30, número 3, págs. 529-532; 1998; ISSN: 0120-0534. Recuperado de:

http://www.redalyc.org/articulo.oa?id=80530309

Robbins, P. (2004). Comportamiento Organizacional. 10ª Edición. México: Editorial Pearson Prentice Hall.

Vroom (1979). Leadership decision-making: an empirical test of the Vroom and Vetton model. Journal of Magement Studies, 16(1), 1-115. 


\section{Humberto Coromoto Peña Rivas}

\section{e-mail: hpenari@upse.edu.ec; hcpr100271@gmail.com}

Nace en San Cristóbal estado Táchira, Venezuela, el 10

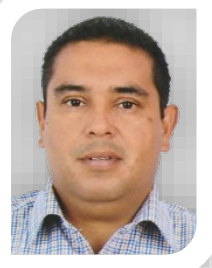
de febrero de 1971; de profesión Ingeniero de Petróleo. Con estudios de Postgrado a nivel de maestría en Gerencia Empresarial; Doctor en Gerencia Avanzada. Egresado de la Universidad Fermín Toro, Venezuela. Experiencia profesional en áreas gerenciales, administrativas y de Ingeniería. Actualmente desempeñándose como Coordinador de investigaciones científicas en la Facultad de Ingeniería de Petróleo de la Universidad Estatal Península de Santa Elena (UPSE); Docente Universitario de la Facultad de Ingeniería de Petróleo; Docente Universitario de la Facultad de Ingeniería Agropecuaria de la Universidad Estatal Península de Santa Elena (UPSE), Ecuador. 


\section{Sabina Gisella Villón Perero}

\section{e-mail: svillonp@upse.edu.ec; sabinagisella@hotmail.es}

Nacida en el Cantón la Libertad, Provincia de Santa

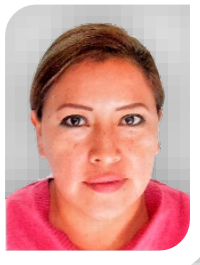

Elena, Ecuador, Magister en Finanzas y Proyectos Corporativos, Universidad Estatal de Guayaquil; Diplomado en Pedagogía de la Educación Técnica y Profesional, Instituto Superior Héctor Pineda ZaldívarCuba y UPSE; Ingeniera Comercial, Universidad Estatal de Guayaquil; experiencia profesional en áreas administrativa, financiera y contable; Docente titular de la Universidad Estatal Península de Santa Elena; Facilitadora Corporación Financiera Nacional: Módulo de Finanzas a los microempresarios de las comunidades de la provincia de Santa Elena (2010); miembro del actual Directorio de la Asociación de Profesores de la Universidad Estatal Península de Santa Elena.

El contenido de este manuscrito se difunde bajo una Licencia de Creative Commons Reconocimiento- 